\begin{tabular}{|l|l|l|l|}
\hline Eiszeitalter u. Gegenwart & $\mathbf{2 8}$ & $\begin{array}{c}167-178 \\
4 \mathrm{Abb} .\end{array}$ & Öbringen/Württ. 1978 \\
\hline
\end{tabular}

\title{
Das Ca/Mg-Verhältnis in den Marschböden, eine Folge postsedimentärer Faktoren
}

\author{
Werner Prange *) \\ Soil science, Northfriesland, marshes, $\mathrm{Ca} / \mathrm{Mg}$-ratio, discussion syn-sedimentary versus \\ post-sedimentary factors.
}

$\mathrm{K}$ u r z f a s s u n g: Das Ca/Mg-Verhältnis wurde auf typischen Kartenausschnitten einer großflächigen geologisch-bodenkundlichen Kartierung auf dem nordfriesischen Festland mit anderen Merkmalen dargestellt und verglichen. Es zeigt keine Beziehungen zu den Sedimentationsbedingungen und damit keine deutbaren Zusammenhänge mit den Kartiereinheiten und den meisten Geländemerkmalen. Dagegen kommt eine deutliche Abhängigkeit von postsedimentären Faktoren heraus, die sich überall anders überlagern und das $\mathrm{Ca} / \mathrm{Mg}$-Verhältnis bestimmen. Es ist deshalb als Grundlage für eine Kartierung und Klassifizierung der Marschböden nicht geeignet.

\section{[Post-sedimentary processes as controlling factors of the $\mathrm{Ca} / \mathrm{Mg}$-ratio]}

A bstract: The $\mathrm{Ca} / \mathrm{Mg}$-ratio, together with other data drawn in typical maps of a large scale soil mapping on the North Frisian mainland, are compared with each other. The $\mathrm{Ca} / \mathrm{Mg}-$ ratio indicate no relations with the conditions under sedimentation and therefore no explainable connections with the soil units and most of the other field characteristics. On contrary there is a clear dependence from post-sedimentary factors, which everywhere add in an other kind and determine the $\mathrm{Ca} / \mathrm{Mg}$-ratio. Therefore it is not suitable as basis for mapping and classifying marsh soils.

\section{Einführung}

Nach dem Ca/Mg-Verhältnis wurden die Marschen in Niedersachsen in See-, Brackund Flußmarschen untergliedert (Müller 1958, 1964, 1970), in der Annahme, der heutige Ionenbelag und vor allem das $\mathrm{Ca} / \mathrm{Mg}-$ Verhältnis seien durch den Salzgehalt des Wassers bei der Sedimentation und den ursprünglichen Kalkgehalt der abgelagerten Sedimente geprägt worden.

$\mathrm{Da}$ auf dem nordfriesischen Festland die Marschen der älteren vor dem Geestrand liegenden Köge (Abb. 1) geologisch, archäologisch und bodenkundlich nach im Gelände faßbaren Merkmalen kartiert und durch Laborergebnisse ergänzt worden waren, kann an diesem Material geprüft werden, welche Methode am besten für die Kartierung und Klassifizierung der Marschböden geeignet ist. Die geologischen Ergebnisse, bereits im Zusammenhang mit der Landschafts- und Siedlungsgeschichte dargestellt (PRANGE 1963, 1967 a und b, 1968, 1969), bilden die Grundlage dieser Untersuchung.

Zunächst waren die zahlreichen geologischen und bodenkundlichen Merkmale mit Hilfe statistischer Methoden miteinander und mit den im Labor ermittelten Analysendaten verglichen worden (PRANGe \& BRÜMMER \& WeBER 1974).

In der vorliegenden Arbeit werden die $\mathrm{Ca} / \mathrm{Mg}$-Werte auf Karten einiger typischer Gebiete Nordfrieslands anderen Merkmalen gegenübergestellt und aus dem lokalen Zusammenhang heraus interpretiert. Damit lassen sich auch die Ursachen kleinräumiger Unterschiede innerhalb der Kartiereinheiten direkt ablesen, die statistisch nicht herauskommen.

*) Dr. Werner P r a n g e, Geologisch-Paläontologisches Institut und Museum der Universität Kiel, Olshauserstraße 40/60, D-2300 Kiel. 


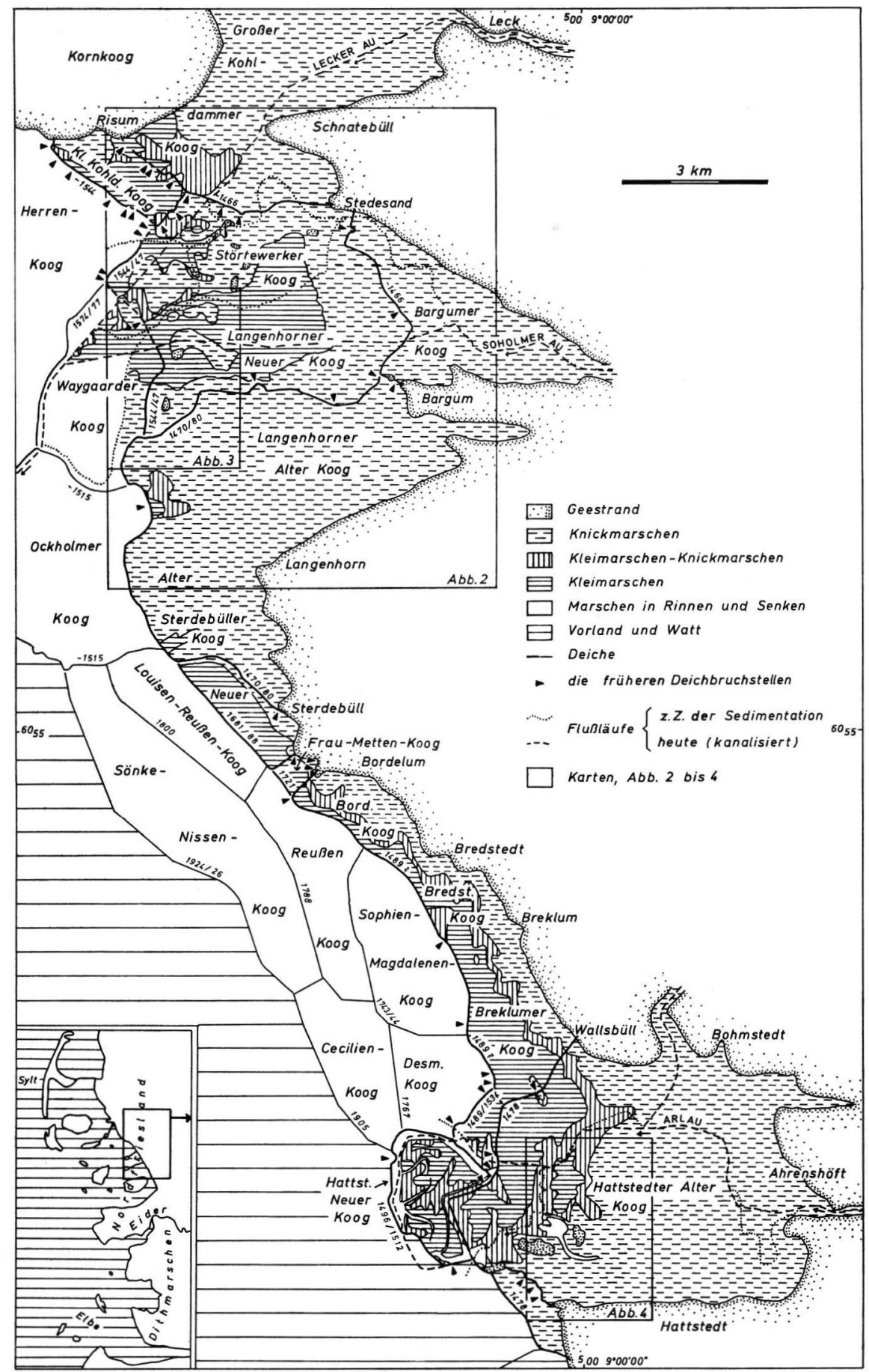

Abb. 1: Geologisch-bodenkundliche Ubersichtskarte der kartierten Köge auf dem nordfriesischen Festland mit der Lage der Kartenausschnitte Abb. 2 bis 4. 
Das verwendete Untersuchungsmaterial, die Geländemethode bei der Kartierung und vor allem die Gesichtspunkte bei der Auswahl der repräsentativen Profile, auf die diese Untersuchungen aufbauen, wurden bereits beschrieben (PrANGE \& Brümmer \& Weber 1974).

\title{
II. Die Ursachen der Unterschiede im Ca/Mg-Verhältnis
}

Das Ca/Mg-Verhältnis, der Quotient aus wasserlöslichem und austauschbarem Ca und $\mathrm{Mg}$, wird von mehreren Faktoren bestimmt. Deshalb werden besonders anhand von Karten die Beziehungen der $\mathrm{Ca} / \mathrm{Mg}$-Werte zu den Kartiereinheiten, der Korngrößenverteilung, dem Carbonatgehalt, den $\mathrm{pH}$-Werten, der Höhenlage der Geländeoberfläche zu NN und dem Salzwassereinfluß untersucht (Abb. 2 bis 4).

\author{
A. Die Beziehungen des Ca/Mg-Verhältnisses \\ $\mathrm{zu}$ anderen Faktoren
}

\section{Süßwasserzuflüsse}

Der Salzgehalt des Meerwassers wird an der schleswig-holsteinischen Westküste nur örtlich etwas durch Süßwasser erniedrigt, da von dem schmalen Landrücken außer der Eider nur kleine Auen zufließen.

Das ergibt auch eine Mengenberechnung nach den Pegeln Soholm und Dörpum für das Einzugsgebiet der Soholmer Au (Landesamt für Wasserhaushalt und Küsten SchleswigHolstein in Kiel 1973). Demnach flossen im Jahre 1972 etwa $5,1 \mathrm{~m}^{3} / \mathrm{sec}$ in die Soholmer $\mathrm{Au}$ ab. Das entspricht während einer Flut in sechs Stunden rechnerisch $110160 \mathrm{~m}^{3}$. Gleichzeitig würden auf das Marschgebiet an der Soholmer Au von etwa $40 \mathrm{~km}^{2}$ mit einer durchschnittlichen NN-Höhe der Geländeoberfläche und bei einer Überflutungshöhe von $1 \mathrm{~m}$ etwa 40 Mill. $\mathrm{m}^{3}$ Salzwasser auflaufen. Dividiert man die Salzwassermenge durch das in gleicher Zeit dazukommende Süßwasser, so macht das nur etwa den 360. Teil des Salzwassers aus, fällt also bei einem Salzgehalt von etwa $25 \%$ vor den heutigen Deichen praktisch nicht ins Gewicht.

Auch wenn man nur die mittleren, die der Au am nächsten gelegenen Marschen berücksichtigt, die etwa $20 \mathrm{~km}^{2}$ groß sind, oder wenn man eine Überflutung von nur einem halben Meter annimmt, hat das zulaufende Süßwasser keinen wesentlichen Einfluß. Ahnlich fällt die Berechnung für die Lecker Au, deren Pegel bei Leck nur einen Abfluß von $2,25 \mathrm{~m}^{3} / \mathrm{sec}$ verzeichnete, und für die Arlau aus.

Das Süßwasser überströmt das schwerere Salzwasser, in dem sich am Grunde das Sediment ablagert. Die Vermischung (vgl. auch BECKMANN 1971) ist gering und hängt von Windrichtung und -stärke sowie von der Morphologie unter dem Wasser ab und ändert sich damit täglich, ohne für frühere Zeiten im einzelnen rekonstruierbar zu sein.

Der Salzgehalt des Wassers am Geestrand, in dem sich die stark tonigen Sedimente ablagerten, kann also nicht 2 bis 3\%o betragen haben, wie nach MüLLER's Sedimentationskonzept (1964) angenommen wurde, und ist deshalb nicht die Ursache für die überwiegend niedrigen $\mathrm{Ca} / \mathrm{Mg}$-Werte in den Knickmarschen am Geestrand. So läßt ein Vergleich der $\mathrm{Ca} / \mathrm{Mg}$-Werte in den Knickmarschen der alten Köge vor Bredstedt, in die kaum Süßwasser zufließt (Abb. 1), mit denen in den Marschen beiderseits der Soholmer und Lecker Au (Abb. 2) keine generellen Unterschiede erkennen. Auch kleinräumiger, wie im Gebiet der Soholmer und Lecker Au (Abb. 2), spiegelt sich das bei der Sedimentation nur örtlich durch die Auen zufließende Süßwasser nicht im heutigen $\mathrm{Ca} / \mathrm{Mg}$-Verhältnis wider. 
Die Knickmarschen Nordfrieslands sind zwar wie in Niedersachsen aufgebaut und haben einen ähnlichen Ionenbelag, müssen aber in Nordfriesland im Meerwasser mit wenig erniedrigtem Salzgehalt abgelagert worden sein. Das hatten auch geologisch-archäologische Untersuchungen aufgrund der mittelalterlichen Salztorfgewinnung ergeben (PRANGE $1971 \mathrm{c}$ ).

Auch wenn das zufließende Süßwasser z.Z. der Sedimentation einen nennenswerten Einfluß auf den Salzgehalt gehabt hätte, wäre das $\mathrm{Ca} / \mathrm{Mg}$-Verhältnis dadurch nicht erniedrigt worden. Da Süßwasser im Verhältnis zum Meerwasser mehr $\mathrm{Ca}^{2+}$ - als $\mathrm{Mg}^{2+}$ Ionen zuführt, müßte es zu einer Erhöhung der $\mathrm{Ca} / \mathrm{Mg}$-Quotienten gekommen sein. Das

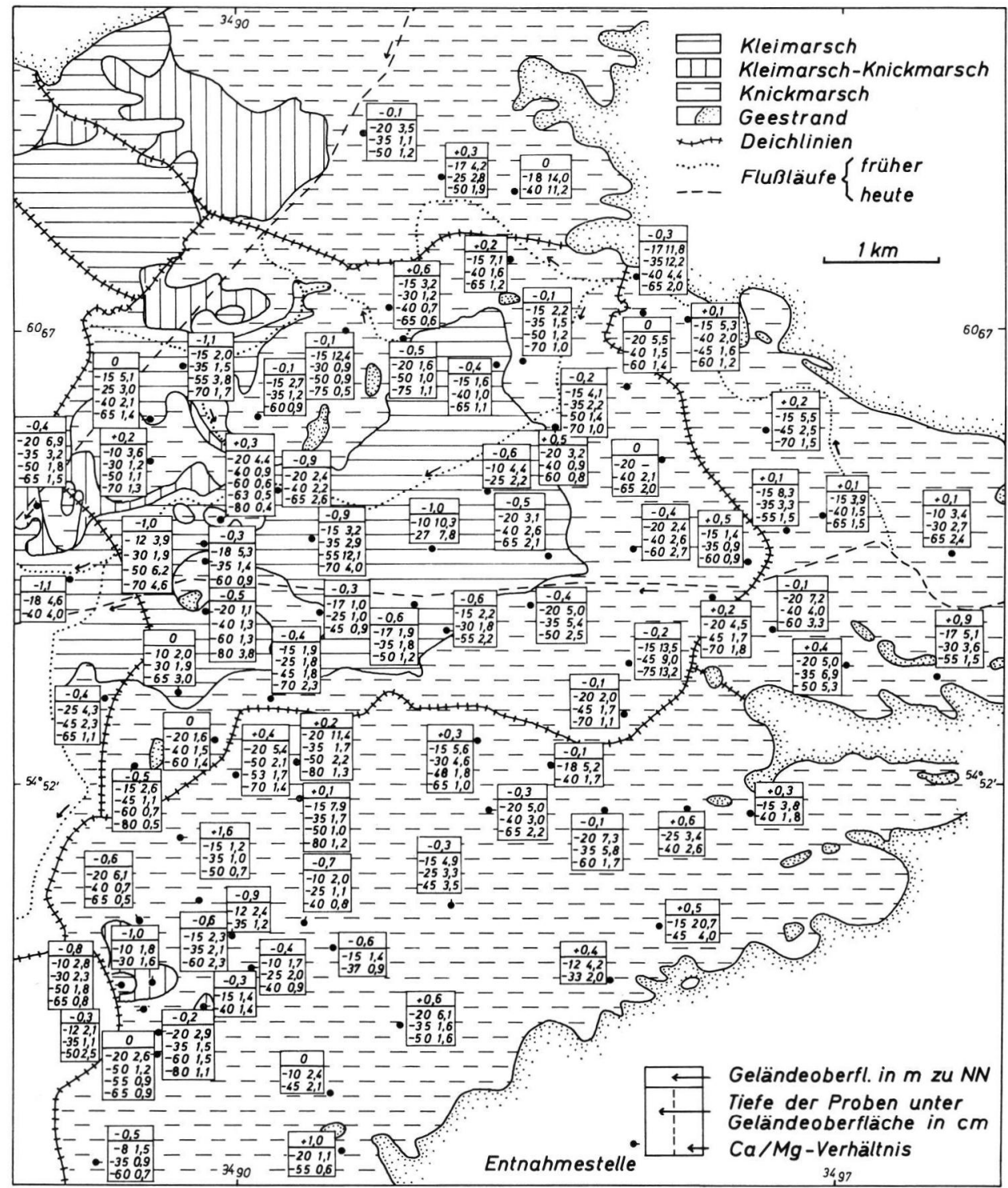

Abb. 2: Das $\mathrm{Ca} / \mathrm{Mg}$-Verhältnis in den Marschen an der Soholmer und Lecker Au im Zusammenhang mit den Kartiereinheiten und der Höhenlage der Profile zu NN. 
war postsedimentär in den niedrigen, nahe am Geestrand gelegenen Knickmarschen der Fall, die bis vor etwa 25 Jahren viele Monate im Jahr von den Auen her überschwemmt waren. Dadurch wurden die dort höheren, zwischen 2 und 6 liegenden $\mathrm{Ca} / \mathrm{Mg}$-Werte verursacht (Abb. 4). Sie sind damit deutlich höher als die überwiegend unter 2 liegenden Werte in den weiter seewärtigen niedrigen Gebieten sowie in den höher gelegenen Knickmarschen (Abb. 3 u. 4).

\section{Kartiereinheiten}

Vergleicht man die $\mathrm{Ca} / \mathrm{Mg}$-Werte zwischen den Kleimarschen, Kleimarschen-Knickmarschen und Knickmarschen, so lassen sich auf allen Karten keine generellen Unterschiede erkennen (Abb. 2 bis 4 ).

In den Oberböden schwanken die $\mathrm{Ca} / \mathrm{Mg}$-Quotienten sehr; daran ist die verschieden starke Düngung abzulesen. Stellenweise scheint sie sehr gering zu sein oder zu fehlen, da die $\mathrm{Ca} / \mathrm{Mg}$-Quotienten nicht wesentlich von denen der darunterfolgenden Horizonte abweichen. In jenen zeigen sich Unterschiede von Profil zu Profil, die aber keinen Zusammenhang mit den Kartiereinheiten erkennen lassen, obwohl diese etwa auch den Sedimentationszonen entsprechen. Somit können die $\mathrm{Ca} / \mathrm{Mg}$-Werte nicht durch primäre Faktoren bei der Sedimentation verursacht sein.

Enst die statistische Untersuchung des Gesamtmaterials hatte geringe Unterschiede in den $\mathrm{Ca} / \mathrm{Mg}$-Quotienten zwischen den Kleimarschen, Kleimarschen-Knickmarschen und Knickmarschen ergeben (Prange \& BrüMmer \& Weber 1974). Sie passen aber nicht zu dem Sedimentationskonzept MülleR's (1964), nach dem man die niedrigsten Werte in den Knickmarschen erwarten müßte. Sie liegen vielmehr in den nicht verdichteten Kleimarschen. Nur in der Kartiereinheit der niedrig gelegenen Böden in Rinnen und Senken, in denen das $\mathrm{Ca} / \mathrm{Mg}-$ Verhältnis stark vom salzhaltigen Grundwasser beeinflußt wird, sind sie noch niedriger.

Auch die Art der Schichtung, die am besten die Sedimentationsbedingungen bzw. die Faziesgebiete charakterisiert, zeigt statistisch gesehen keine gesicherten Zusammenhänge zum $\mathrm{Ca} / \mathrm{Mg}$-Verhältnis: die Werte zwischen den geschichteten Kleimarschen und den ungeschichteten Knickmarschen unterscheiden sich nicht signifikant.

\section{Korngrößenverteilung}

Ein Vergleich der Tongehalte mit den $\mathrm{Ca} / \mathrm{Mg}$-Quotienten deuten sich ebenfalls keine generellen Beziehungen an (Abb. 3 und 4).

Die Tongehalte schwanken auch innerhalb der Kartiereinheiten. Am Beispiel des südlichen Geestrandes im Arlau-Gebiet (Abb.4) zeigt sich, daß die Tongehalte in den Knickmarschen, abgesehen vom Geestrand mit anthropogenen Sandbeimengungen, von über $50 \%$ nahe am Geestrand bis auf etwas über 30\% weiter westlich abnehmen. Die örtlichen Unterschiede zwischen gleichweit vom Geestrand entfernten Profilen hängen mit kleinen Höhenunterschieden bei der Sedimentation zusammen. Sie steuerten die Wege des sedimentanliefernden Wassers und damit auch die feinen Korngrößenunterschiede. Sie gehen aber nicht mit Änderungen in den $\mathrm{Ca} / \mathrm{Mg}$-Quotienten einher (Abb. 4).

An dem zweiten Beispiel, dem geestferneren Gebiet an der Soholmer und Lecker Au (Abb. 3), liegen die Flächen der Kartiereinheiten nicht geestrandparallel. Vielmehr werden die Tongehalte und damit die Kartiereinheiten besonders durch kleinräumige Höhenunterschiede und die Lage zu den Rinnen bestimmt. So liegen im N Knickmarschen mit hohem Tongehalt im Schutz einer sichelförmigen Erhebung, die den Westrand einer ehemaligen Hallig inmitten von tiefen Rinnen darstellt. Das inselförmige Knickmarschgebiet in der 


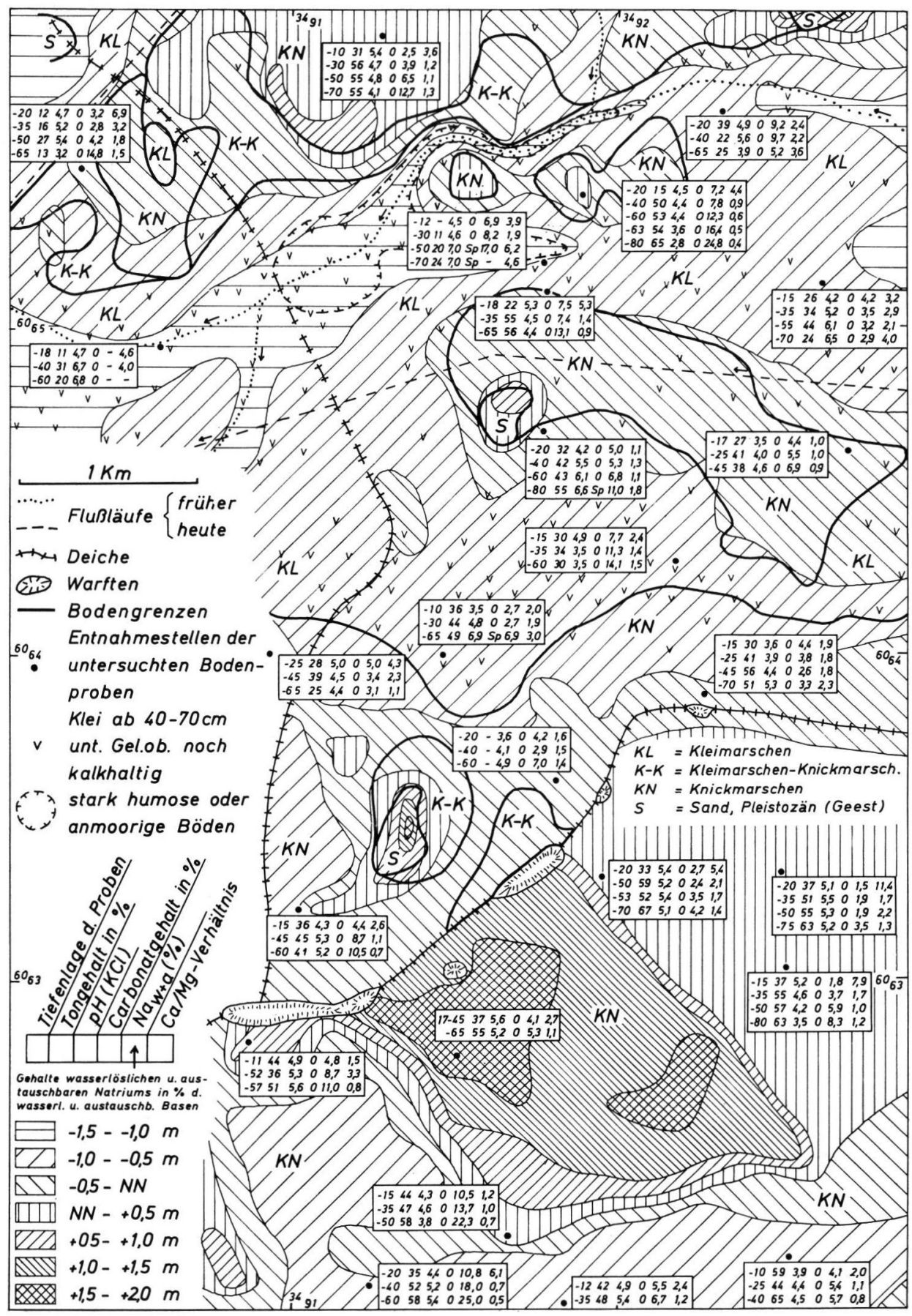

Abb. 3: Das Ca/Mg-Verhältnis in den Marschen an der unteren Soholmer und Lecker $\mathrm{Au}$ im $\mathrm{Zu}$ sammenhang mit der NN-Höhe der Geländeoberfläche, der Tiefenlage der Proben, dem Tongehalt und $\mathrm{pH}$-Wert sowie dem Carbonat- und $\mathrm{Na}-$ Gehalt. 
Mitte der Karte hat sich fahnenartig im Lee einer kleinen Geestaufragung gebildet. In den Kleimarschen im Gebiet der weiten Niederungen nimmt der Tongehalt zu den Rändern allgemein zu und ebenfalls landeinwärts. Somit ist es kein Wunder, daß bei diesen allgemeinen Übergängen, besonders in Gebieten ohne deutliche morphologische Unterschiede, fließende Übergänge sein müssen, bei denen die Zuordnung des Bodens zu einer Kartiereinheit auf der Zusammenfassung von Tongehalt, Schichtung und Verdichtung beruht.

Auch in diesem Gebiet mit einem verwickelteren geologischen Aufbau zeigen die Ca/ Mg-Quotienten keinen Zusammenhang mit den Kartiereinheiten (Abb. 3). Statistisch bestehen nur signifikante Unterschiede zum Tongehalt, die u. a. mit der Silikatverwitterung zusammenhängen (vgl. Prange \& Brümmer \& Weber 1974, Schroeder \& Brümmer 1969).

\section{Carbonatgehalt}

Im größten Teil der kartierten alten Köge Nordfrieslands ist der Klei bis über $70 \mathrm{~cm}$ unter Geländeoberfläche und damit am Geestrand großflächig ganz entkalkt.

Entgegen der Annahme Müller's (1964, 1970), der stark tonige Klei der Knickmarschen sei kalkarm oder -frei sedimentiert worden, war er einst kalkhaltig wie heute in den jüngsten Kögen. Das beweisen Sedimentfallen mit kalkhaltigem muschelreichen Klei (zugeschlickte Salztorfstiche) und Muschelabdrücke im Gebiet des ganz entkalkten Kleis (PRANGe 1971 c). Wie sollte sich der Kalkgehalt auch bei der Korngrößensortierung im Watt nur in der gröberen Fraktion ansammeln, da doch Kalkpartikelchen in allen Größen vorliegen.

Stellenweise sind im Unterboden ab $40 \mathrm{~cm}$ unter Geländeoberfläche noch Reste des natürlichen Kalkgehaltes erhalten. Diese Gebiete fallen deutlich mit höheren $\mathrm{Ca} / \mathrm{Mg}$-Werten in den Karten auf. Das trifft einerseits für den Sterdebüller Neuen Koog und den FrauMetten-Koog zu (Abb. 1), die später als die anderen untersuchten Köge eingedeicht worden waren (PrANGe 1968, 1971 b), so daß die Böden noch nicht so tief entkalkt sind. Aber auch in den tiefliegenden Teilen der älteren, um 1500 eingedeichten Köge findet man im Unterboden noch Reste des Kalkgehaltes, wie in einigen schmalen, an der heutigen Oberfläche etwa $1 \mathrm{~m}$ tiefen Prielen im Arlau-Gebiet. In den weiten nicht verlandeten Niederungen an der Soholmer und Lecker $\mathrm{Au}$ ist das in den Kleimarschen unter NN, überwiegend unter $-0,5 \mathrm{~m} \mathrm{NN}$ der Fall (Abb. 3). Diese Gebiete waren noch bis vor 25 Jahren viele Monate im Jahr überschwemmt, so daß die Carbonate nicht so tief ausgelaugt werden konnten. Die von Profil zu Profil etwas unterschiedliche Entkalkungstiefe, die sich z. T. in der Verteilung der Signaturen der Abb. 3 andeutet (Kalkgehalt noch oberhalb oder erst unterhalb von $70 \mathrm{~cm}$ unter Geländeoberfläche beginnend), erklärt sich dadurch, daß auch bei gleichem Tongehalt und gleicher Geländehöhe die Schichtung und damit die Verteilung der Tone verschieden ist. Damit ändert sich auch die Durchlässigkeit. Zum anderen ist auch der primäre Kalkgehalt vor allem in den Rinnen verschieden.

\section{5. $\mathrm{pH}-\mathrm{Wert}$}

Die Zusammenhänge, die sich beim Vergleich der $\mathrm{Ca} / \mathrm{Mg}$-Werte mit den Carbonatgehalten ergeben hatten, kommen auch in den $\mathrm{pH}$-Werten heraus, da zwischen beiden enge Beziehungen bestehen.

Abgesehen von den Oberböden, deren $\mathrm{pH}$-Werte überall verschieden stark von der Düngung bestimmt werden, bestätigt die Abb. 4 den statistischen Befund, daß sich die Kleimarschen, Kleimarschen-Knickmarschen und Knickmarschen in den $\mathrm{pH}(\mathrm{KCl})$-Werten kaum unterscheiden (4,7 bis 4,9). Das zeigt auch die Abb. 3, obwohl dort Korngrößenzusammensetzung und Höhenlage von Profil zu Profil sehr verschieden sind. Nur im S der 


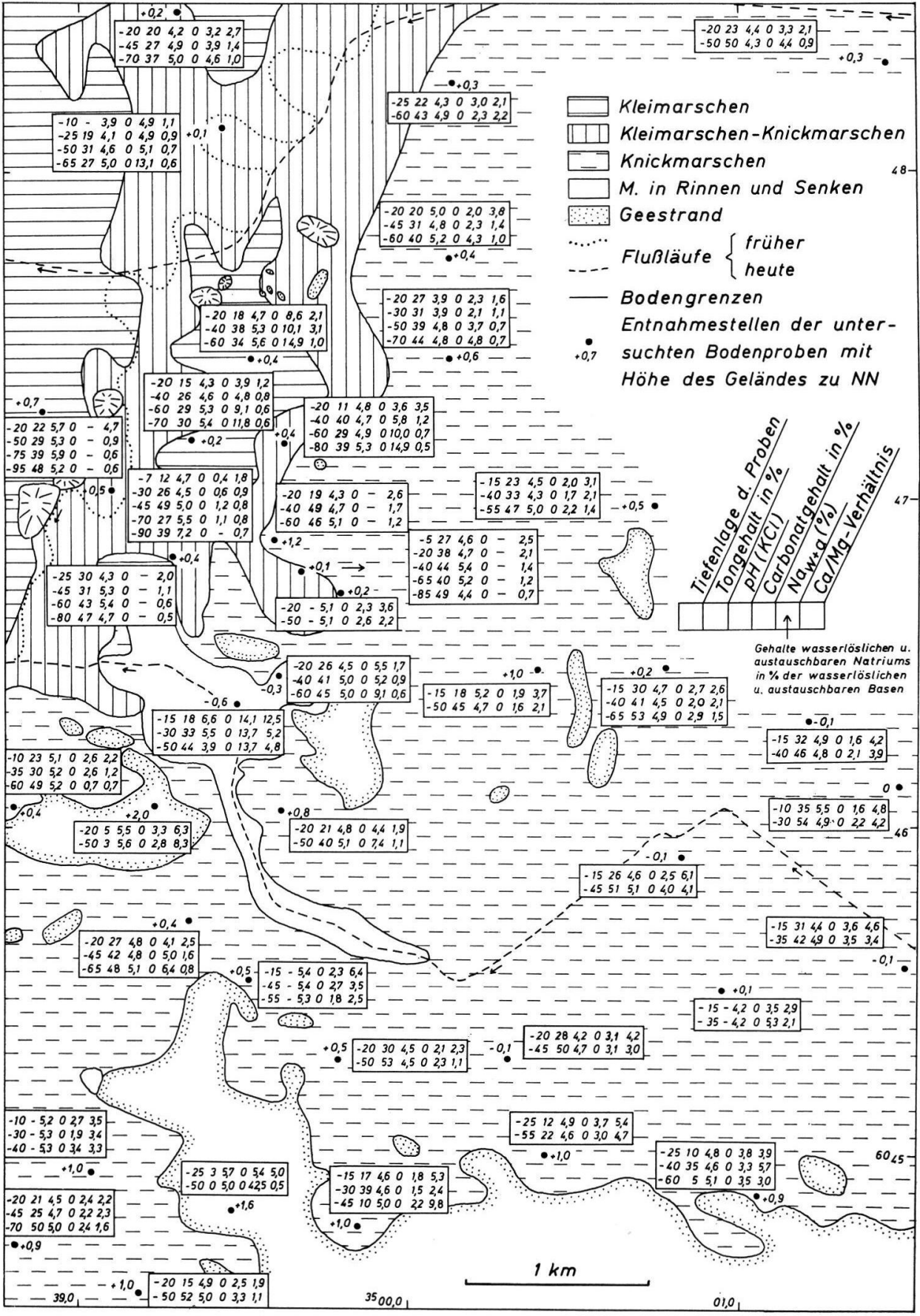

Abb. 4: Das Ca/Mg-Verhältnis in den Marschen im S des Arlau-Gebietes im Zusammenhang mit der Tiefenlage der Proben, dem Tongehalt und pH-Wert sowie dem Carbonat- und Na-Gehalt. 
Arlau-Marschen (Abb. 4), wo die geestrandparallelen Zonen verschiedener Tongehalte bzw. Kartiereinheiten deutlicher ausgeprägt sind, zeigen die Kleimarschen und KleimarschenKnickmarschen im NW mit überwiegend über 5 etwas höhere $\mathrm{pH}(\mathrm{KCl})$-Werte als die Knickmarschen mit unter 5 im SE. Das hängt mit Unterschieden in der Höhenlage und im Tongehalt zusammen, die einen Einfluß auf den Grad der Versauerung haben.

In den Rinnen und Senken, in denen hohes Grundwasser und Überschwemmungen die Entkalkung und Versauerung verzögerten, steigen die $\mathrm{pH}(\mathrm{KCl})$-Werte nach unten an und liegen statistisch für die Kartiereinheit der Rinnen und Senken bei 5,9. Auch das weist wie bei den Carbonaten auf eine Abhängigkeit von der Höhenlage der Marsch und damit von der Entwässerung hin, die sich auch im $\mathrm{Ca} / \mathrm{Mg}$-Verhältnis abzeichnet.

\section{Salzhaltiges Grundwasser}

Der Salzgehalt im Klei pendelt sich wenige Jahre nach der Eindeichung auf ein Gleichgewicht ein. Es unterliegt Schwankungen, die mit Unterschieden in den Niederschlägen und der Verdunstung sowie der Grundwasserhöhe und -versalzung zusammenhängen.

Die heutigen prozentualen Anteile an wasserlöslichen und austauschbaren NatriumIonen an der Summe der austauschbaren Basen $\left(\mathrm{Na}_{\mathrm{w}+\mathrm{a}} \%\right)$ weisen besonders auf eine der Ursachen hin, die die Höhe des $\mathrm{Ca} / \mathrm{Mg}$-Verhältnisses bestimmen: mit den Na-Ionen des Salzwassers werden auch $\mathrm{Mg}$-Ionen zugeführt, die das $\mathrm{Ca} / \mathrm{Mg}-\mathrm{Verhältnis} \mathrm{erniedrigen.}$

Allgemein nehmen die $\mathrm{Na}_{\mathrm{W}+\mathrm{a}} \%$-Werte in den Profilen nach unten $\mathrm{zu}$, die $\mathrm{Ca} / \mathrm{Mg}$ Quotienten ab (Abb. 3 und 4). Das ist in allen Gebieten verschiedener Kartiereinheiten der Fall; sie unterscheiden sich auch statistisch nicht darin (PRANGE \& BRÜMMER \& Weber 1974). Daß diese Zunahme der Natrium-Werte auf den Einfluß salzhaltigen Grundwassers zurückgeht und damit auch von der Höhenlage der Marschoberfläche bzw. der Entwässerung abhängt (vgl. auch Dittmer 1954, Dümmler 1970, Ludwig \& Barkhausen \& Streif 1973, Schreitling 1959), läßt sich in den Böden der Niederungen ablesen (Abb. 3). Dort steigen die $\mathrm{Na}$-Werte deutlich rascher an; die $\mathrm{Ca} / \mathrm{Mg}$-Werte sind besonders niedrig. Das deutet sich auch in der Senke der Abb. 4 an: schon im Oberboden sind die $\mathrm{Na}_{\mathrm{w}}+\mathrm{a} \%$-Werte höher als in den Unterböden der hochliegenden Marschen rundherum. Nur an solchen niedrigen Stellen findet man im Gebiet der Soholmer und Lecker Au eine Salzvegetation (CHRISTIANSEN \& STEINBERg 1956).

Der Salzwassereinfluß hängt jedoch auch örtlich mit der Entfernung der Profile vom Meer zusammen. Am Südrand der Abb. 3 liegen drei Profile in etwa gleicher Höhenlage, bei denen nur im westlichen die Na-Werte nach unten stark zunehmen. Dieser Zusammenhang kommt auch auf Abb. 4 heraus. Am Geestrand dagegen (Abb. 4) dürften die Na-Gehalte auf die Salze aus den Niederschlägen und Aerosolen zurückgehen (vgl. auch ScHulz 1972), zumal die $\mathrm{Na}_{\mathrm{w}+\mathrm{a}} \%$ - Werte unmittelbar am Geestrand nach unten kaum noch zunehmen oder sogar abnehmen. So wird das $\mathrm{Na}$ nicht aus salzhaltigem Grundwasser stammen, denn gerade an dieser Stelle fließt von der höheren Geest her etwas Süßwasser zu.

Andererseits kommen jedoch in der Marsch Süß- und Salzwasser nebeneinander vor, ohne daß immer ein Zusammenhang mit der Lage zum Meer festzustellen wäre. Das geht auch aus Angaben der Bauern über die Brunnen auf den pleistozänen Sandaufragungen in der Marsch hervor. So ist z. B. das Grundwasser auf dem Geestvorsprung der Abb. 4 stärker versalzen als auf der weiter im NW vorgelagerten Geestinsel, an deren Rand die $\mathrm{Na}_{+} \mathrm{a} \%$-Werte im Oberboden höher sind als in tieferen Horizonten.

Die Unterschiede in der Versalzung der einzelnen Gebiete werden durch die wechselnden Höhenverhältnisse und die Schichtfolge mitbestimmt (vgl. auch DitTmer 1956). So bestehen überall andere Verbindungen zu den Wasserleitern auf der Geest und unter den Marschenablagerungen, mächtige Kleischichten können salzhaltiges Grundwasser nach oben 
abschirmen und Torfschichten lassen leicht Salzwasser bis in die geestrandnahen Hinterländer der Marsch durch.

Die Wege von Süß- und Salzwasser lassen sich im einzelnen kaum feststellen und somit auch nicht die $\mathrm{Ca}-$ und $\mathrm{Mg}$-Zufuhr, die das $\mathrm{Ca} / \mathrm{Mg}$-Verhältnis mitbestimmen. Es kann deshalb kein Merkmal zur Kartierung und Klassifizierung der Marschböden sein.

\section{B. Die Überlagerung verschiedener Einflüsse auf das $\mathrm{Ca} / \mathrm{Mg}-\mathrm{Verhältnis}$}

Da mehrere Faktoren die Höhe des $\mathrm{Ca} / \mathrm{Mg}$-Verhältnisses bestimmen, überlagern sie sich in ihrer Einzelwirkung überall anders.

Der Einfluß des Kalkgehaltes in niedrigen Gebieten (Abb. 3) wird durch den des salzhaltigen Grundwassers überlagert, so daß die wechselnde Höhe der $\mathrm{Ca} / \mathrm{Mg}$-Quotienten neben anderen Einflüssen auch das Verhältnis dieser beiden Faktoren anzeigt. So findet man in der Senke der Abb. 4 trotz hoher $\mathrm{Na}_{\mathrm{w}+\mathrm{a}} \%$ - Werte auch ein hohes $\mathrm{Ca} / \mathrm{Mg}$-Verhältnis, da noch Reste des Kalkgehaltes erhalten sind. In etwas höherer Lage am Rande der Senke ist der Boden dagegen schon ganz entkalkt, so daß der Einfluß des salzhaltigen Grundwassers im niedrigeren $\mathrm{Ca} / \mathrm{Mg}$-Verhältnis deutlich zu erkennen ist

In den weiten Gebieten mit entkalkten Böden ohne größeren Einfluß salzhaltigen Grundwassers und mit einheitlich niedrigen $\mathrm{Ca} / \mathrm{Mg}$-Werten zeigt sich, daß sie in starkem Maße auf salzhaltige Niederschläge und Aerosole zurückgehen, die mehrere Jahrhunderte lang gleichmäßig einwirken konnten.

In den besonders niedrig gelegenen entkalkten Knickmarschen in den Buchten am Geestrand haben jahrhundertelange Süßwasserüberschwemmungen, die durch weitere Vordeichungen und Sackungen infolge zunehmender Entwässerung noch verstärkt wurden, so viel $\mathrm{Ca}$ zugeführt, daß der Einfluß des $\mathrm{Mg}$ aus salzhaltigen Niederschlägen und Aerosolen zurückgedrängt wurde. So findet man dort deutlich höhere $\mathrm{Ca} / \mathrm{Mg}$-Quotienten als in den höher gelegenen Knickmarschen (vgl. Abb. 4).

Salzwassereinbrüche infolge von Deichbrüchen (vgl. Abb. 1 und Prange 1971 a) führten stellenweise wieder $\mathrm{Mg} \mathrm{zu}$ und veränderten das Gleichgewicht zwischen den anderen Faktoren.

Auch heute wird das $\mathrm{Ca} / \mathrm{Mg}$-Verhältnis weiter verändert. So muß die stärkere Entwässerung eine schnellere und tiefere Entkalkung bewirken. Der Meeresspiegelanstieg, vor allem aber die zunehmende Sackung und der Süßwasserentzug bei der Entwässerung lassen das salzhaltige Grundwasser weiter landeinwärts vordringen (vgl. auch SCHREITLING 1959, vaN VeEN 1956). Mit der intensiveren Nutzung der entwässerten Marschböden geht auch eine stärkere Düngung einher, die sich besonders auf das $\mathrm{Ca} / \mathrm{Mg}$-Verhältnis in den Oberböden auswirken wird.

\section{Ergebnisse}

Die Ca/Mg-Quotienten wurden zusammen mit einigen anderen Merkmalen auf Karten typischer Marschgebiete dargestellt. Die regionalen Unterschiede und der Vergleich dieser Daten lassen erkennen, daß das $\mathrm{Ca} / \mathrm{Mg}$-Verhältnis kaum von den Faktoren bei der Ablagerung der Sedimente bestimmt wurde, wie nach dem Sedimentationskonzept Müllers (1964) angenommen worden war; denn es bestehen keine Beziehungen zu den Kartiereinheiten bzw. Sedimentationszonen, dem Salzgehalt des Wassers bei der Sedimentation und dem ursprünglichen Kalkgehalt der Schlicke. Auch statistisch hatten sich keine Zusammenhänge gezeigt, außer mit dem Tongehalt, von dem die Mg-Zufuhr z. T. gespeist wird (Prange \& Brümmer \& Weber 1974). 
Das $\mathrm{Ca} / \mathrm{Mg}$-Verhältnis erweist sich eindeutig als ein Kennwert, der erst postsedimentär im Laufe der Boden- und Landschaftsgeschichte durch das Zusammenspiel vieler Faktoren geprägt und immer wieder verändert wurde. Das sind vor allem Entsalzung, Entkalkung, Versauerung und Silikatverwitterung, die mit der Entwässerung, der dadurch begünstigten Sackung sowie dem Vordringen salzhaltigen Grundwassers einhergehen. Hinzu kommen die jahrhundertelangen salzhaltigen Niederschläge und Aerosole, bei Deichbrüchen Salzwassereinbrüche sowie in niedrigen Hinterländern Süßwasserüberschwemmungen. In jüngster Zeit verstärkt sich der Einfluß der Düngung. Die Ergebnisse bestätigen die geologischarchäologischen und bodenkundlichen Untersuchungen von BRÜMMER (1968), BRÜMMER \& Schroeder (1968), Brümmer \& Schroeder \& Mitarbeiter (1971), Dümmler (1974), Prange (1971 c), Schroeder (1969) und Schroeder \& Brümmer (1968, 1969).

Der Vergleich der $\mathrm{Ca} / \mathrm{Mg}$-Quotienten mit den anderen Merkmalen auf den Karten zeigt deutlich, daß der Einfluß jeder dieser Faktoren schon auf kleinstem Raum verschieden ist, so daß die Überlagerung überall zu einem anderen $\mathrm{Ca} / \mathrm{Mg}$-Verhältnis führen muß. Es ist deshalb nicht als Grundlage für eine Kartierung und Klassifizierung der Böden in der Marsch geeignet.

\section{Schriftenverzeichnis}

Beckmann, H. (1971): Die Salinitätsverhältnisse in den Zuflüssen der Bucht von Sada (NW-Spanien, Galizien). - Lehrstuhl f. Erdölgeologie der T. U. Clausthal, 69 S., 19 Abb., Clausthal.

Brümmer, G. (1968): Untersuchungen zur Genese der Marschen. - Diss. Univ. Kiel, 350 S., 34 Abb., 12 Fot., 47 Tab.; Kiel.

- \& Schroeder, D. (1968): Prozesse der Marschgenese. - Mitt. dt. bodenkundl. Ges., 8: 247250; Göttingen.

- \& Schroeder, D. \& Mitarbeiter (1971): Landschaften und Böden Schleswig-Holsteins - insbesondere Böden der Marschlandschaft. - Mitt. dt. bodenkundl. Ges., 13: 7-57, 3 Abb., 1 Karte; Göttingen.

Christiansen, W. \& Steinberg, K. (1956): Binnenland-Salzwiesen der nordfriesischen Marsch. Die Küste, 5: 103-112, 5 Abb., 2 Tab.; Heide/Holst.

Ditrmer, E. (1954): Der Mensch als geologischer Faktor an der Nordseeküste. - Eiszeitalter u. Gegenwart, 4/5: 210-215; Öhringen/Württ.

- (1956): Die Versalzung des Grundwassers an der schleswig-holsteinischen Westküste. - Die Küste, 5: 87-102, 3 Abb.; Heide/Holst.

DÜMmLeR, H. (1970): Der Einfluß von salzhaltigem Grundwasser auf die Kationenbelegung nordfriesischer Marschböden. - Mitt. dt. bodenkundl. Ges., 10: 302-305, 5 Abb., 2 Tab.; Göttingen.

- (1974): Anwendung von geologisch-bodenkundlichen Stoffverteilungsmustern bei der Darstellung und Interpretation der Kationenverteilung einer Marschlandschaft (Schleswig-Holstein). — Mitt. dt. bodenkundl. Ges., 18: 305-312, 5 Abb.; Göttingen.

Landesamt f. Wasserhaushalt u. Küsten Schleswig-Holstein in Kiel (1973): Deutsches Gewässerkundliches Jahrbuch. Küstengebiet der Nord- und Ostsee. Abflußjahr 1972. - 158 S., 1 Karte; Kiel.

Ludwig, G., Barkhausen, J. \& Streif, H. (1973): Geochemische Untersuchungen über Paläosalinität und Ablagerungsraum holozäner Küstensedimente Ostfrieslands. - Geol. Jb., D 3: 35-54, 12 Abb., 3 Tab.; Hannover.

MüLLER, W. (1958): Grundsätzliche Betrachtungen zur systematischen Gliederung der Marschböden. - Geol. Jb., 76: 11-24; Hannover.

- (1964): Unterschiede in den chemischen und physikalischen Eigenschaften von fluviatilen, brakkischen und marinen Sedimenten. - Developments in Sedimentology, 1: 293-300, 2 Abb.; Amsterdam, London, New York (Elsevier).

- (1970): Zur Genese und Klassifizierung der Marschböden. - Mitt. dt. bodenkundl. Ges., 10: 294-301; Göttingen. 
Prange, W. (1963): Das Holozän und seine Datierung in den Marschen des Arlau-Gebietes, Nordfriesland. - Meyniana, 13: 47-76, 11 Abb.; Kiel.

- (1967 a): Uber die Beziehungen zwischen Schichtfolge und Meeresspiegelanstieg im Holozän der Nordseemarschen. - Geol. Rdsch., 56: 709-726, 6 Abb.; Stuttgart.

- (1967 b): Geologie des Holozäns in den Marschen des nordfriesischen Festlandes. - Meyniana, 17: 45-94, 20 Abb., 2 Taf., 1 Tab.; Kiel.

- (1968): Geologische Untersuchungen in den Marschen der alten Köge vor Bredstedt, Nordfriesland. - N. Jb. Geol. Paläont., Mh., (1968): 619-640, 5 Abb.; Stuttgart.

- (1969): Alte mündliche Überlieferung aus Nordfriesland und ihre Verwendung bei geologischen und siedlungsgeschichtlichen Untersuchungen in der Marsch. - Nordfries. Jb., N. F., 4/5: 262-277, 3 Abb.; Bredstedt.

- (1971 a): Geologisch-historische Untersuchungen an Deichbrüchen des 15. bis 17. Jahrhunderts in Nordfriesland. - Nordfries. Jb., N. F., 7: 25-55, 8 Abb.; Bredstedt.

- (1971 b): 250 Jahre Frau-Metten-Koog. Eine geologisch-historische Darstellung seiner Entstehung. - Nordfriesland, 20: 179-184, 2 Abb.; Bredstedt.

- (1971 c): Geologisch-archäologische Untersuchungen zu den Entstehungsbedingungen der Marschen in Nordfriesland. - Z. Pflanzenernähr. u. Bodenk., 130: 151-160, 2 Abb.; Weinheim/ Bergstr.

- , BRÜMmer, G. \& Weber, E. (1974): Untersuchungen zur Kartierung und Klassifizierung der Böden in der Marsch. - Meyniana, 25: 59-86, 5 Abb., 7 Tab.; Kiel.

SchreITLING, K.-T. (1959): Beiträge zur Erklärung der Salzvegetation in den nordfriesischen Kögen. - Mitt. Arbeitsgemeinsch. Floristik Schleswig-Holstein u. Hamburg, 8: 3-98, 45 Abb., 16 Tab.; Kiel.

Schroeder, D. (1969): Bodenkunde in Stichworten. - 145 S., 53 Abb., 22 Tab., 2 Taf.; Kiel (F. Hirth).

- \& Brümmer, G. (1968): Zur Genese und Klassifizierung der Marschen. - Mitt. dt. bodenkundl. Ges., 8: 243-245; Göttingen.

- \& BrümmeR, G. (1969): Beiträge zur Genese und Klassifizierung der Marschen. I. Problematik der Marschengenese und -klassifizierung und Untersuchungen zum $\mathrm{Ca} / \mathrm{Mg}$-Verhältnis. $-\mathrm{Z}$. Pflanzenernähr. u. Bodenk., 122: 228-249, 4 Abb., 4 Tab.; Weinheim/Bergstr.

Schulz, H. D. (1972): Grundwasserneubildungen berechnet aus der Chlorid-Bilanz. - Geol. Mitt., 12: $53-60,3 \mathrm{Abb}$.; Aachen.

VeEn, J. VAN (1956): Die Versalzung der niederländischen Marschen und ihre Bekämpfung. - Die Küste, 5: 73-86, 11 Abb.; Heide/Holst. 